\title{
Serum uric acid levels in pregnancy induced hypertension preeclampsia
}

\author{
Ajay Kumar Singh ${ }^{1}$, Raj Kumar ${ }^{2, *}$, Vinay Kumar Singh ${ }^{3}$, Saurabh Srivastava ${ }^{4}$, Ashish Sharma ${ }^{5}$ \\ ${ }^{1}$ Assistant Professor, ${ }^{5}$ Tutor, Dept. of Biochemistry, MRA Medical College Ambedkar Nagar Uttar Pradesh, ${ }^{2}$ Associate \\ Professor, Dept. of Biochemistry, BRD Medical College, Gorakhpur, Uttar Pradesh, ${ }^{3}$ Assistant Professor, PIDS, GIDA, \\ Gorakhpur, Uttar Pradesh, ${ }^{4}$ Post Doctor Fellow, Centre for Interdisciplinary Research in Basic Sciences, Jamia Millia Islamia (A \\ Central University), New Delhi, India
}

*Corresponding Author:

Email: akhandrajk@gmail.com

Received: $1^{\text {st }}$ February, 2018

Accepted: $20^{\text {th }}$ April, 2018

\begin{abstract}
Preeclampsia is a pregnancy specific syndrome which involves an increase of arterial blood pressure, accompanied by proteinuria, oedma or even both. This investigation was intended to compare the serum uric acid level in preeclamptic and normal pregnant women and its correlation with some important maternal and fetal results. The subjects were partitioned into two groups, Group I: control subjects normal pregnant women and Group II: pregnancy induced hypertensive women. Each groups consisted of 30 pregnant women of Uttar Pradesh (India). The blood sample was collected and analyzed for biochemical parameters, blood urea, serum uric acid, serum creatinine and total protein. The result data were dissected by using SPSS. The systolic (SBP) and diastolic blood pressure (DBP) and serum uric acid were significantly $(\mathrm{P}<0.05)$ increased in group II subjects as compared to group I subjects. Other parameters like urea, creatinine and total protein were statically significant $(\mathrm{P}<0.01,0.05)$ in group II subjects as compared to group I subjects. Maternal serum uric acid concentration along with other parameters like urea, creatinine and total protein are significantly increased from early pregnancy and increased more rapidly across pregnancy in women who arise preeclampsia.
\end{abstract}

Keywords: Preeclampsia, Uric acid, Early-onset preeclampsia, Late-onset preeclampsia.

\section{Introduction}

Pre-eclampsia (PE) is a well known obstetric disease, involves different system and organs with unknown etiology. ${ }^{1}$ It is a pregnancy particular disorder of pregnant females which includes rise of SBP, joined by proteinuria, oedma or even both. Preeclampsia influences about $2-8 \%$ of all pregnancies and a several types of complications have been reported with this disease. $^{2,3}$

$\mathrm{PE}$ is a disorder, known as the beginning of hypertension and proteinuria following 20 weeks of incubation in already normotensive and non-protinuric women. The mechanism of disorder is known, but new emerging consensus says the complex polygenetic trait in which maternal and fetal genes as well as environmental factors are involved. ${ }^{4}$ The multisystem issue of obscure etiology can likewise be characterized by development of hypertension to degree of 140/90 $\mathrm{mmHg}$ or more with proteinuria instigated by $20^{\text {th }}$ weeks after pregnancy. ${ }^{5}$

Hypertensive disorders are important causes of premature delivery, IUGR and intrauterine fetal death. It accounts for $15-20 \%$ of maternal death in developing as well as developed nations. ${ }^{6,7}$ An increased uric acid level generally precedes the beginning of hypertension and proteinuria in preeclampsia. ${ }^{8}$ More first revealed over than 80 years prior, expended serum uric acid is another regular clinical finding in females with clinically obvious preeclampsia. ${ }^{9}$

Uric acid is a well known marker of tissue injury, oxidative stress, and renal dysfunction. ${ }^{10-13}$ Uric acid produced at the end of purine metabolism and synthesized in the presence of xanthine oxidase enzyme. The factors like hypoxia, ischemia of the placenta and cytokines such as interferon induced the expression of xanthine oxidase. ${ }^{11,14,15}$

The raised level of uric acid has been shown to cause specific adverse affects on blood pressure in studies of non-pregnant rats. ${ }^{12,16,17}$ Thus, increased uric acid is a marker of severity in preeclampsia. If this is the case, then uric acid should be increased before the syndrome is clinically evident. ${ }^{12-14}$ Hyperuricemia is one of the earliest and generally steady observations noted in preeclamptic pregnancies. While elevated concentrations of circulating uric acid are not uniformly seen in every woman with preeclampsia, they do appear to identify a subset of preeclamptic women who are at greater risk for maternal and fetal morbidities. ${ }^{8,16}$ This examination was planned to compare the values of serum uric acid in preeclamptic and normal pregnant females and its relationship with some vital maternal and fetal results.

\section{Materials and Methods}

Study Group: The present examination was completed on total 60 women from outpatient department of Government medical college Ambedkar Nagar (India). Out of which 30 were normal pregnant women (Group I) and 30 were pre-eclamptic patient (group II) was divided. The investigation was directed in the department of Biochemistry and physiology of Government medical college Ambedkar Nagar Uttar Pradesh (India). The 
Group II further divided into two groups as earlybeginning preeclampsia (EOPE) and late- beginning preeclampsia (LOPE) based on their time of diagnose either before or after 34 gestational weeks.

Recording of Systemic Arterial Blood Pressure: BP was measured in supine position by utilizing mercury sphygmomanometer. Systolic blood pressure (SBP) and diastolic blood pressure (DBP) of all the subjects were recorded.

Selection of Cases: Criteria for selection of the subjects in this examination were as follows-

1. No previous history of hypertension.

2. No history of urinary tract infection.

3. Absence of any other medical complications (cardiovascular disease, renal disease, collagen vascular disease) affiliated with preeclampsia.

Inclusion Criteria: Age 21-36 years, primigravida, 2840 weeks gestations and absence of labor contraction. Informed consent was taken. The preeclampsia was defined as BP always greater than $140 / 90 \mathrm{mmHg}$ and proteinuria above $0.3 \mathrm{gm} / 24$ hour.

The blood sample was collected in plain vial and analyzed for biochemical investigations which include (1) uric acid, (2) Urea, (3) Creatinine and (4) Total protein.

Biochemical Investigations: Blood sample was collected in plain vial and incubated at $37^{\circ} \mathrm{C}$ for 30 minutes, after incubation, clot was removed and remaining sample was taken in centrifuge test tube, test sample were centrifuged at $3000 \mathrm{rpm}$ for 10 to 20 minutes. Supernatant was collected in clean and dry serum test tube for analysis of uric acid, urea, creatinine and total protein. Biochemical parameter, uric acid was estimated by spectrophotometer in a commercial available kit (Enzymatic-colorimetric trinder methods). Urea was estimated by semi autoanalyser (EnzymaticUV kinetic method). Creatinine and total protein were estimated by colorimeter (Jaffe kinetic, Biuret reaction method) according to manufacturer's instruction.

\section{Statistical Analysis}

The comparisons between two groups were analyzed by student's t-test. All parameters were given as mean \pm standard deviation. The criterion for significance was $p<0.05$. Pearson's correlation was used to evaluate the correlations between the variables. Data analysis was performed with the statistical package for the social sciences version 16.00 (SPSS, Chicago, ielinosis USA).

\section{Results}

The study group of 60 women included 30 normotensive (group I) and rest 30 with pregnancy-induced hypertension (group II) of different severity. Table No.1 Showing the comparative changes of physiological parameters in group I and group II. All of the investigated subjects were in the range of 21-36 years age group. The mean age of the group I was $27.40 \pm 3.55$ and $27.03 \pm 3.91$ of group II. In the present examination, we have divided the all samples in the two groups, Group I (normal) and Group II (PE). The demographic data is briefly summarized in Table 1. In the terms of age and BMI, there were no significant differences were observed. The Mean value of SBP and DBP are significantly increase $(p<0.05)$ in group II than group I (Table 1).

There were no critical contrasts between the gatherings I and II regarding age, BMI. The Mean estimation of SBP and DBP are altogether increment $(\mathrm{p}<0.05)$ in preeclampsia gathering (PE) than in the control gathering (Table 1).

The biochemical parameters like uric acid, urea level, creatinine and total protein of group I and Group II were analyzed and summarized in Table 2 . There were significant differences were found between these groups (I and II) in the terms of uric acid, urea, creatinine and total protein. The estimation mean value of uric acid was significantly higher in group II (7.63 \pm 0.59$)$ compared with Group I $(4.94 \pm 0.83)$. The significant increase in urea level (Group I: $26.34 \pm 4.78$ and group II: $46.55 \pm$ 3.31), creatinine level (Group I: $0.73 \pm 0.14$ and group II: $1.16 \pm 0.19$ ), total protein level (Group I: $6.86 \pm 0.45$ and group II: $5.22 \pm 0.54$ ) were found.

The preeclampsia has divided in two groups; earlyonset preeclampsia (EOSP) and late-onset preeclampsia (LOSP). The biochemical parameters were analyses in group I and EOSP, LOSP of group II. On the account of uric acid, there is significant increased level of uric acid in $\operatorname{EOSP}(8.63 \pm 0.97)$ from the LOSP $(4.94 \pm 0.83)$. The level uric acid of LOSP $(7.62 \pm 0.65)$ get slightly decreases from the EOSP, but still, significantly high from the group I. The urea concentration of EOSP $(46.53 \pm 3.55)$ and LOSP $(46.56 \pm 3.11)$ non-significantly correlate each other (Table 3), but significantly decrease in the comparison of Group I. The creatinine level of EOSP $(1.19 \pm 0.19)$ and LOSP $(1.14 \pm 0.20)$ shows non-significant correlation (Table 3 ). The total protein level of EOSP $(6.10 \pm 1.04)$ shows significantly increases with LOSP (5.28 \pm 0.50$)$.

Table 1: The comparative changes of physiological parameters in group I and group II subjects of study

\begin{tabular}{|c|c|c|c|c|c|}
\hline Groups & & Age & BMI & SBP & DBP \\
\hline \multirow{4}{*}{ Group I } & Min & 21.00 & 19.02 & 100 & 70 \\
& Max & 34.00 & 24.21 & 130 & 90 \\
\cline { 2 - 6 } & Mean \pm SD & $27.40 \pm 3.55$ & $21.85 \pm 1.58$ & $114.37 \pm 0.29$ & $76.56 \pm 7.75$ \\
& Min & 21.00 & 19.73 & 120 & 80 \\
Group II & Max & 36.00 & 24.84 & 150 & 110 \\
\hline & Mean \pm SD & $27.03 \pm 3.91^{\mathrm{NS}}$ & $22.64 \pm 1.70^{\mathrm{NS}}$ & $138.12 \pm 8.81^{*}$ & $76.56 \pm 9.00^{*}$ \\
\hline
\end{tabular}

*Significant at $0.05(\mathrm{p}<0.05) ;{ }^{\text {Ns }}$ Non Significant 
Table 2: The comparative changes of biochemical parameters in group I and group II subjects of study

\begin{tabular}{|c|c|c|c|c|c|}
\hline Groups & & Uric Acid & Urea & Creatinine & Total protein \\
\hline \multirow{4}{*}{ Group I I } & 3.50 & 18.00 & 0.52 & 6.09 \\
& Max & 6.30 & 34.00 & 0.9 & 7.79 \\
\cline { 2 - 6 } & Mean \pm SD & $4.94 \pm 0.83$ & $26.34 \pm 4.78$ & $0.73 \pm 0.10$ & $6.86 \pm 0.45$ \\
\hline \multirow{3}{*}{ Group II } & Min & 6.54 & 40.02 & 0.91 & 4.29 \\
\cline { 2 - 6 } & Max & 8.4 & 51.02 & 1.58 & 6.04 \\
\hline & SE SD & $7.63 \pm 0.59^{*}$ & $46.55 \pm 3.31^{*}$ & $1.16 \pm 0.19^{* *}$ & $5.22 \pm 0.54^{*}$ \\
\hline
\end{tabular}

$* *$ Highly Significant at $0.01(\mathrm{p}<0.01)$

*Significant at $0.05(\mathrm{p}<0.05)$

Table 3: The comparative changes of biochemical parameters in LOSP and EOSP in group II subjects of study

\begin{tabular}{|c|c|c|c|c|c|}
\hline Groups & & Uric Acid & Urea & Creatinine & Total protein \\
\hline & Min & 6.54 & 42.33 & 0.91 & 4.53 \\
LOSP & Max & 08.4 & 51.02 & 1.58 & 6.01 \\
\cline { 2 - 6 }$(\mathbf{n}=\mathbf{1 3})$ & Mean \pm SD & $7.62 \pm 0.65$ & $46.56 \pm 3.11$ & $1.14 \pm 0.20$ & $5.28 \pm 0.50$ \\
\hline & Min & 7.27 & 40.02 & 0.98 & 4.29 \\
EOSP & Max & 09.9 & 50.45 & 1.56 & 7.86 \\
\cline { 2 - 6 }$(\mathbf{n}=\mathbf{1 7})$ & Mean \pm SD & $8.63 \pm 0.97^{*}$ & $46.53 \pm 3.55^{\mathrm{NS}}$ & $1.19 \pm 0.19^{\mathrm{NS}}$ & $6.10 \pm 1.04^{* *}$ \\
\hline & SE & 0.31 & 1.18 & 0.06 & 0.28 \\
\hline
\end{tabular}

$* *$ Highly Significant at $0.01(\mathrm{p}<0.01)$

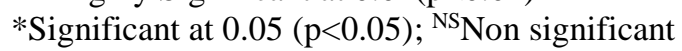

\section{Discussion}

There is many biochemical tests have reported as an indicator of the preeclampsia development, including maternal serum uric acid. ${ }^{3}$ In the present study, we showed for the first time the correlation between uric acid, urea levels, creatinine and total protein content of both groups. Moreover, we demonstrated that connection between's both systolic and diastolic pressure as in preeclampsia than in the control group. There is no significant correlation of age and BMI were found between control and preeclampsia groups.

In our examination, altogether increment of uric acid level was found in preeclampsia (Table 2). There are couple of previous investigation has also suggested that the uric acid level in preeclampsia significantly increased. ${ }^{15-19}$ Here, we have found that, the uric acid level of EOSP was significantly higher than LOSP. Uric acid has antioxidant properties as well as it contributes as free radical scavenger in human serum. ${ }^{20}$ The examined increased level of uric acid might happens due to defensive reaction restricts unsafe impacts of free radical action and oxidative stress. ${ }^{11,18}$ The urea level was found significantly decrease in preeclampsia, but no correlation observed between LOSP and EOSP. The same pattern was observed in the creatinine level also.

The level of uric acid depends up on various factors like altered kidney function as well as enzymatic defects in purine metabolism. ${ }^{21}$ During pregnancy, level uric acid level reduced initially because of numerous factors like impacts of estrogen and increased glomerular filtration rate (GFR). ${ }^{22}$ However, the level of uric acid slowly increases in non-pregnant women with time of gestation. ${ }^{12,16}$

\section{Conclusion}

The present study showed that, the uric acid in PE women was slightly higher than normal pregnant women but, significant correlation was found between EOSP and LOSP group of women. The urea and creatinine level was found higher in PE women than normal pregnant women but, there was no significant correlation found between EOSP and LOSP women. These findings support that correlation of biochemical parameters with Preeclampsia as well as EOSP and LOSP.

Conflicts of Interest: No conflict of interest has declared.

\section{Acknowledgments}

This study was supported in part by the Department of Biochemistry and Gynaecology at BRD Medical College Gorakhpur, Uttar Pradesh and MRA Medical College Ambedkar Nagar, Uttar Pradesh, India.

\section{References}

1. Kaypour F, Masomi Rad H \& Ranjbar Novin N, The predictive value of serum uric acid, roll-over test, and body mass index in pre-eclampsia. Int J Gynaecol Obstet. 2006;92:133-134.

2. Cnossen JS, Ruyter-Hanhijarvi H, Post JA, Mol BW, Khan KS \& Riet G, Accuracy of serum uric acid determination in predicting pre-eclampsia: a systematic review. Acta Obstet Gynecol Scand. 2006;85:519-525. 
3. Taefi A, Sadat JA \& Human Delavari, The Role of Serum Uric Acid in Preeclampsia. Journal of Family and Reproductive Health. 2008;2:159-162.

4. Weerasekera DS \& Peiris H, The significance of serum uric acid, creatinine and urinary microprotein levels in predicting pre-eclampsia. J Obstet Gynaecol. 2003;23:1719.

5. Berhan Y, No Hypertensive Disorder of Pregnancy; No Preeclampsia-eclampsia; No Gestational Hypertension, No Hellp Syndrome, Vascular Disorder of Pregnancy. Ethiop J Health Sci. 2016;26:177-186.

6. Duley L, The global impact of pre-eclampsia and eclampsia. Semin Perinatol. 2009;33:130-137.

7. Jeyabalan A, Epidemiology of preeclampsia: Impact of obesity. Nutr Rev. 2014;71:1-13.

8. Shannon AB \& Roberts JM, Uric Acid as a Pathogenic Factor in Preeclampsia. Placenta. 2008;29:67-72.

9. Redman CW, Beilin LJ, Bonnar J \& Wilkinson RH, Plasma-urate measurements in predicting fetal death in hypertensive pregnancy. Lancet. 1976;26:1370-1373.

10. Kyaw A, A simple colorimetric method for ascorbic acid determination in blood plasma. Clin Chim Acta. 1978;86:153-157.

11. Kharb S. Uric acid and ascorbic acid levels in pregnancy with preeclampsia and diabetes. Webmed central Biochemistry. 2010;1:718.

12. Powers RW, Bodnar LM, Ness RB, Cooper BS, Marcia JG, Michael PF, Daftary AR \& , Uric acid concentrations in early pregnancy among preeclamptic women with gestational hyperuricemia at delivery. Am J Obstet Gynecol. 2006;194(1)160.

13. Maged AM, Aid G, Bassiouny N, Eldin DS, Dahab S \& Ghamry NK, Association of biochemical markers with the severity of pre-eclampsia. Int J Gynaecol Obstet. 2017; 136:138-144.

14. Nobumoto E, Masuyama H, Maki J, Eguchi T, Tamada S, Mitsui T, Eto E, Hayata K \& Hiramatsu Y, Comparison of Kidney Function between Gestational Hypertension and Preeclampsia. Acta Med Okayama. 2017;71:161-169.
15. Hickman PE, Michael CA \& Potter JM, Serum uric acid as a marker of pregnancy-induced hypertension. Aust $N Z$ J Obstet Gynaecol. 1982;22:198-202.

16. Osakwe CR, Ikpeze OC, Ezebialu IU, Osakwe JO $\&$ Mbadugha NN, The predictive value of serum uric acid for the occurrence, severity and outcomes of preeclampsia among parturients at nnewi Nigeria. Niger J Med. 2015;24(3):192-200.

17. Mazzali M, Hughes J, Kim YG, Jefferson JA, Kang DH, Gordon KL, Lan HY, Kivlighn S \& Johnson RJ, Elevated uric acid increases blood pressure in the rat by a novel crystal-independent mechanism. Hypertension. 2001;38(5)1101-1106.

18. Kharb S, Kaur R, Singh V \& Sangwan K, Birth weight, cord blood lipoprotein and apolipoprotein levels in Indian newborns. Int J Prev Med. 2010;1(1)129-33.

19. Many A, Hubel CA \& Roberts JM, Hyperuricemia and xanthine oxidase in preeclampsia. Am J Obstet Gynecol. 1996;174:288-291.

20. Waring WS, Webb DJ \& Maxwell SR, Systemic uric acid administration increases serum antioxidant capacity in healthy volunteers. J Cardiovasc Pharmacol. 2001;38:(3)365-371.

21. Johnson RJ, Kang DH, Feig D, Kivlighn S, Kanellis J, Watanabe S, Tuttle KR, Rodriguez-Iturbe B, HerreraAcosta J \& Mazzali M, Is there a pathogenetic role for uric acid in hypertension and cardiovascular and renal disease. Hypertension. 2003;41(6):1183-1190.

22. Carter J \& Child A, Serum uric acid levels in normal pregnancy. Aust N.Z.J Obstet Gynaecol. 1989;29:313314.

How to cite this article: Singh AK, Kumar R, Singh VK, Srivastava S, Sharma A. Serum uric acid levels in pregnancy induced hypertension preeclampsia. Int $\mathrm{J}$ Clin Biochem Res. 2018;5(3):365-368. 